УДК 517.956

\author{
V. M. Kyrylych, O. Z. Slyusarchuk
}

\title{
BOUNDARY VALUE PROBLEMS WITH NONLOCAL CONDITIONS FOR HYPERBOLIC SYSTEMS OF EQUATIONS WITH TWO INDEPENDENT VARIABLES
}

\begin{abstract}
V. M. Kyrylych, O. Z. Slyusarchuk. Boundary value problems with nonlocal conditions for hyperbolic systems of equations with two independent variables, Mat. Stud. 53 (2020), 159-180.

Nonlocal boundary value problems for arbitrary order hyperbolic systems with one spatial variable are considered. A priori estimates for general nonlocal mixed problems for systems with smooth and piecewise smooth coefficients are obtained. The correct solvability of such problems is proved. Examples of additional conditions necessity are provided.
\end{abstract}

1. Introduction. Theory of boundary-value problems for hyperbolic partial differential equations with two independent variables is almost half a century old. However, even today, it offers new problems solving of which require various methods of modern analysis. Such interest is caused by the fact that these equations arise in the studying of important processes of science and technology

Not limited to the well-known examples of string oscillations, electromagnetic perturbations, etc., let us point to more complex processes that lead to equations and systems of hyperbolic type.

A basic evolution model of age-structured population $([38,65])$ leads to the Lotka-MacKendrick system

$$
\partial_{t} u+\partial_{x} u=-\lambda(x) u, \quad u(x, 0)=\nu(x), \quad u(0, t)=v(t) \int_{0}^{L} \beta(x) u(x, t) d x
$$

where $\lambda, \nu, L, \beta$ are standard biological parameters, and the birth rate $v(t)$ is often used to control the population ( $v$ is the control).

Similar problems arise in solid-state physics ([15]). The peculiarity of these problems is that the characteristics of hyperbolic system are both inclined and horizontal. From a physics point of view, this means that some of perturbations in the medium propagate with a finite velocity and some with unbounded one. The mathematical model of such a problem takes the form

$$
\frac{\partial u}{\partial t}+\operatorname{sh} E \frac{\partial u}{\partial x}=-u^{2} \operatorname{ch} E, \quad \frac{\partial E}{\partial x}=p u, \quad \frac{\partial \varphi}{\partial x}=-q E .
$$

2020 Mathematics Subject Classification: 35L50.

Keywords: nonlocal boundary value problem; hyperbolic system; method of characteristics; piecewise smooth coefficients; Volterra-type integral equation; piecewise smooth solution.

doi:10.30970/ms.53.2.159-180

(C) V. M. Kyrylych, O. Z. Slyusarchuk, 2020 
Here $u$ is the concentration of impurity sputtering of an electroluminophore; $E$ is the electric field strength; $\varphi$ is the potential; $p, q$ are some constants.

The initial and boundary conditions for this system are set as follows

$$
\begin{gathered}
u(x, 0)=u_{0}(x), \quad u(0, t)-\frac{\partial u}{\partial x}(0, t)=0, \quad u(l(t), t)=g(l(t)), \\
E(l(t), t)=0, \quad \varphi(0, t)=\varphi_{0}, \quad \varphi(l(t), t)=0
\end{gathered}
$$

where $l(t)$ is a currently unknown curve describing the position of the area right boundary and satisfying the relation

$$
l^{\prime}(t) l(t) g(l(t))-b \int_{0}^{l(t)} u(x, t) \operatorname{sh} E d x=0
$$

and the initial condition

$$
\int_{0}^{l(0)} x g(x) d x=-\frac{\varphi_{0}}{p q} .
$$

Many applications, such as the theory of service networks with complex routing, require that the solvability conditions of some infinite differential equation systems must be find out [25]. In the hyperbolic case, the general formulation of the mixed problem for a countable system takes the form [17]

$$
\begin{gathered}
\frac{\partial u_{i}}{\partial t}+\lambda_{i}(x, t) \frac{\partial u_{i}}{\partial x}=\sum_{j=1}^{\infty} a_{i j}(x, t) u_{j}+f_{i}(x, t), \quad i \in \mathbb{N}, \\
u_{i}(x, 0)=g_{i}(x), \quad x \in[0, l], \quad i \in \mathbb{N}, \\
u_{i}(0, t)=\sum_{i \in I^{-}} \alpha_{i j}(t) u_{j}(0, t)+h_{i}(t), t \in[0, T], \quad i \in I^{+}, \\
u_{i}(l, t)=\sum_{i \in I^{+}} \beta_{i j}(t) u_{j}(l, t)+h_{i}(t), t \in[0, T], i \in I^{-} .
\end{gathered}
$$

Here $I^{+}=\{2 k-1: k \in \mathbb{N}\}$, and $I^{-}=\mathbb{N} \backslash I^{+}$.

The analysis of elastic oscillations of a piezoelectric converter [67] of thin flat ring form with impulse voltage $V(t)$ applied between the outer and inner radii is based on the study of solutions to the problem

$$
\begin{gathered}
\frac{\partial^{2} u}{\partial t^{2}}=v^{2}\left(\frac{\partial^{2} u}{\partial r^{2}}+\frac{1}{r} \frac{\partial u}{\partial r}-\frac{1}{r} u\right), \quad r_{1}<r<r_{2}, t>0 \\
u(r, 0)=u_{t}^{\prime}(r, 0)=0, \quad u\left(r_{2}, t\right)=0 \\
h \int_{r_{1}}^{r_{2}}\left(\frac{\partial u}{\partial r}-\frac{1}{r} u\right) d r=V(t) .
\end{gathered}
$$

The colliding process of ropes having a uniform connection and moving at the same speed [11] leads to various boundary-value problems for following systems of equations

$$
\frac{\partial^{2} u_{i}}{\partial x \partial y}=f_{i}\left(x, y, u_{1}, u_{2}, \ldots, u_{n}\right), \quad i=1, \ldots, n .
$$


Small vibrations of a beam, taking into account the information about displacements and inertia of rotation, are described by the system [23]

$$
\frac{\partial^{2} u}{\partial x^{2}}-\frac{k \rho}{G} \frac{\partial^{2} u}{\partial t^{2}}=\frac{\partial v}{\partial x}+f(x, t), \quad \frac{\partial^{2} v}{\partial x^{2}}-\frac{\rho}{E} \frac{\partial^{2} v}{\partial t^{2}}=\frac{G F}{K E l}\left(\frac{\partial u}{\partial x}-v\right) .
$$

Here $u$ are the transverse displacements of the beam points; $v$ is the average rotation of the normal section; $G, E$ are the elastic characteristics of the beam; $\rho$ is the linear density; $F$ is the cross-sectional area; $K$ is the geometric characteristic of the system; $l$ is the beam length.

The above examples indicate to the need for the theory of boundary-value problems of differential equations and systems of hyperbolic type with two independent variables such that it would cover both classical problems. For instance, Cauchy problem, mixed problem in rectangular domain, Goursat problem, and new more complex problems, in particular, problems with non-local (discrete or distributed) conditions, problems without initial conditions, problems with moving known or unknown boundaries, etc.

This paper proposes a unified approach to solving nonlocal boundary value problems for linear hyperbolic equations and systems of general form with one spatial variable. It is based on the experience of many scientists who have employed various methods in the study of hyperbolic equations and systems.

The method of characteristics is chosen as a basis of this approach. It allows to reduce the problems under consideration to the corresponding systems of Volterra-type integral equations. Their studying gives us the opportunity to obtain the theorems of existence, uniqueness, and continuous dependence of both classical solutions and different classes of generalized solutions to boundary value problems. Some of results presented here can be obtained by other methods. However, many of the problems considered in this paper are sufficiently comprehensive in the method of characteristics. Such a circumstance justifies the choice of this method as the main one.

In addition, we restrict ourselves to studying only piecewise smooth solutions. Although by means of the results mentioned above, one could significantly extend the solutions class to the problems under consideration by standard methods.

The review is restricted to linear problems, since in this case the theory is largely complete. Cases of other boundary-value problems with detailed literature review, in particular, for nonlinear hyperbolic equations and systems have been considered in $[16,26,32,63]$.

2. Auxiliary statements. Let $l_{1}$ and $l_{2}$ be smooth curves located into the half-plane $t \geqslant 0$ of the plane $x O t, x=a_{1}(t)$ and $x=a_{2}(t)$ its equations respectively, and $a_{1}(0)=a_{1}, a_{2}(0)=a_{2}$, $a_{1}(t)<a_{2}(t), t \in[0, T], T>0$. Denote $G_{\tau}=\left\{(x, t) \in \mathbb{R}^{2}: a_{1}(t)<x<a_{2}(t), 0<t \leqslant \tau\right\}$, where $\tau \in(0, T]$, and $G=G_{T}$. Let us consider the matrix differential equation

$$
\sum_{i=0}^{n} A_{i}\left(x, t, \frac{\partial}{\partial x}, \frac{\partial}{\partial t}\right) u=f(x, t)
$$

of order $n \geqslant 1$ on $G$, where $A_{i}$ is a linear homogeneous differential operator

$$
A_{i}\left(x, t, \frac{\partial}{\partial x}, \frac{\partial}{\partial t}\right) u \equiv \sum_{j=0}^{i} A_{i j}(x, t) \frac{\partial^{i} u}{\partial x^{j} \partial t^{i-j}}
$$

of order $i$. The coefficients $A_{i j}$ are square matrices of order $m \geqslant 1$ with elements $a_{i j}^{p q} \in C^{1}(\bar{G})$; $u$ and $f$ are columns of height $m$ with components $u_{1}, \ldots, u_{m}$ and $f_{1}, \ldots, f_{m}$, respectively. It will be further assumed that $A_{n 0}(x, t) \equiv E_{m}$, where $E_{m}$ is the unit matrix of order $m$. 
Equation (1) is strictly hyperbolic in $G$. That is, the eigenvalue of matrix bundle

$$
A_{n}(x, t, 1, \lambda)=\lambda^{n}+\sum_{j=1}^{n} A_{n j} \lambda^{n-j},
$$

namely the roots $\lambda$ of the characteristic equation

$$
\operatorname{det} A_{n}(x, t, 1, \lambda) \equiv\left|A_{n}(x, t, 1, \lambda)\right|=0,
$$

are real and different for any $(x, t) \in \bar{G}$.

Let us denote these roots by $-\lambda_{1}(x, t), \ldots,-\lambda_{m n}(x, t)$. Suppose a non-zero $m$-dimensional vector $h_{i}=h_{i}(x, t)$ satisfies $A_{n}\left(x, t, 1, \lambda_{i}\right) h_{i}=0$, then it said to be the eigenvector of the matrix bundle $A_{n}$ corresponding to the eigenvalue $\lambda_{i}$ for $i=1, \ldots, m n$.

All eigenvectors $h_{1}, \ldots, h_{m n}$ and eigenvalues $\lambda_{1}, \ldots, \lambda_{m n}$ can be partitioned into $n$ nonintersecting groups of $m$ elements such that the vectors of every group form a base in $\mathbb{R}^{m}$. Denote by $H_{i}=H_{i}(x, t)$ the square matrix of order $m$ such that $h_{(i-1) m+1}, \ldots, h_{i m}$ are its columns and $\Lambda_{i}=\operatorname{diag}\left(\lambda_{(i-1) m+1}, \ldots, \lambda_{i m}\right)$ the diagonal matrix of order $m$. Then $\operatorname{det} H_{i}(x, t) \neq 0$ and $\operatorname{det}\left(\Lambda_{i}-\Lambda_{j}\right) \neq 0$ for any $(x, t) \in \bar{G}$, where $i \neq j$.

Consider the following block matrices

$$
A=A(x, t)=\left(\begin{array}{ccccc}
A_{n 1}(x, t) & A_{n 2}(x, t) & \cdots & A_{n n-1}(x, t) & A_{n n}(x, t) \\
-E_{m} & 0 & \cdots & 0 & 0 \\
\vdots & \vdots & \ddots & \vdots & \vdots \\
0 & 0 & \cdots & -E_{m} & 0
\end{array}\right)
$$

and

$$
P=P(x, t)=\left(\begin{array}{ccc}
H_{1} \Lambda_{1}^{n-1} & \cdots & H_{n} \Lambda_{n}^{n-1} \\
\vdots & \ddots & \vdots \\
H_{1} \Lambda_{1} & \cdots & H_{n} \Lambda_{n} \\
H_{1} & \cdots & H_{n}
\end{array}\right)=\left(P_{i j}\right)_{i, j=1}^{n},
$$

where $P_{i j}=H_{j} \Lambda_{j}^{n-i}$. Because $\operatorname{det}\left(A+\lambda E_{m n}\right)=\operatorname{det} A_{n}(x, t, 1, \lambda)$, the values $-\lambda_{i}(x, t)$ are eigenvalues of the matrix $A$. Therefore, this matrix is similar to a diagonal one. It is easy to see that

$$
\operatorname{det} P(x, t)= \pm \prod_{i=1}^{n} \operatorname{det} H_{i}(x, t) \prod_{1 \leq j<i \leq n} \operatorname{det}\left(\Lambda_{i}(x, t)-\Lambda_{j}(x, t)\right) \neq 0
$$

for any $(x, t) \in \bar{G}$ and $P^{-1} A P=\operatorname{diag}\left(\Lambda_{1}, \ldots, \Lambda_{n}\right)$.

Suppose $P^{-1}=\left(P^{i j}\right)_{i, j=1}^{n}$, where $P^{i j}=P^{i j}(x, t)$ are square matrices of order $m$. Let us introduce the linear homogeneous matrix operators

$$
M_{i} u \equiv M_{i}\left(x, t, \frac{\partial}{\partial x}, \frac{\partial}{\partial t}\right) u \equiv \sum_{j=1}^{n} P^{i j}(x, t) \frac{\partial^{n-1} u}{\partial t^{n-j} \partial x^{j-1}}, \quad i=1, \ldots, n .
$$

It is easy to see that its form a base in the space of linear homogeneous matrix operators of order $n-1$, in particular

$$
\frac{\partial^{n-1} u}{\partial t^{n-i} \partial x^{i-1}} \equiv \sum_{j=1}^{n} P_{i j} M_{j}\left(x, t, \frac{\partial}{\partial x}, \frac{\partial}{\partial t}\right) u, \quad i=1, \ldots, n .
$$

From the above we immediately obtain the decomposition

$$
A_{n}\left(x, t, \frac{\partial}{\partial x}, \frac{\partial}{\partial t}\right) u \equiv \sum_{i=1}^{n} P_{1 i}\left(E_{m} \frac{\partial}{\partial t}+\Lambda_{i} \frac{\partial}{\partial x}\right) M_{i} u+\bar{A}_{n-1}\left(x, t, \frac{\partial}{\partial x}, \frac{\partial}{\partial t}\right) u \text {. }
$$


Here $\bar{A}_{n-1}$ is a linear homogeneous differential operator of order $n-1$, defined in an obvious way, with coefficients depending on the operator $A_{n}$ coefficients and the first derivatives of the matrics $P$ block elements $P_{i j}$.

3. A priori estimates for solutions to general nonlocal mixed problems for systems with smooth coefficients. It is well known about the important role of a priori estimates for solutions in boundary-value theory of hyperbolic-type differential equations $[12,41,68]$. In the case of one spatial variable, the a priori estimates for solutions to general mixed problems of first-order hyperbolic systems by different methods have been obtained in [70-73,78]. The same for one high-order equation have been done in $[10,44,60,61]$. In this section, we mainly use the ideas of $[60,78]$ to obtain a priori estimates for solutions to general mixed problems for system (1).

Suppose system (1) is given on $G ; A_{n j}(x, t) \in C^{1}(\bar{G}), j=1, \ldots, n ; A_{i j}(x, t), f(x, t) \in$ $C(\bar{G}), i=0, \ldots, n-1, j=0, \ldots, i$, and $a_{1}(t), a_{2}(t) \in C^{1}[0, T]$.

Assume that the differences $w_{i}^{1}(t) \equiv \lambda_{i}\left(a_{1}(t), t\right)-a_{1}^{\prime}(t)$ and $w_{i}^{2}(t) \equiv \lambda_{i}\left(a_{2}(t), t\right)-a_{2}^{\prime}(t)$ have no zeros on $[0, T]$. Let us denote by $I_{1}^{+}\left(I_{1}^{-}\right)$the set of indices $i$ such that $w_{i}^{1}(t)>0$ $\left(w_{i}^{1}(t)<0\right)$, and by $I_{2}^{+}\left(I_{2}^{-}\right)$the set of indices $i$ such that $w_{i}^{2}(t)>0\left(w_{i}^{2}(t)<0\right)$.

Suppose $F(x, t)$ is an arbitrary function defined on $G$ and $M$ is a boundary point of $\bar{G}$. Then a value of $F$ at $M$ means the limit value of $F(x, t)$ as $(x, t)$ approaches $M$ by arbitrarily way from $G$.

For (1), we impose the initial conditions

$$
\frac{\partial^{i} u(x, 0)}{\partial t^{i}}=g_{i}(x), \quad i=0,1, \ldots, n-1, \quad x \in\left[a_{1}, a_{2}\right]
$$

and the boundary ones

$$
\left.\sum_{i=1}^{2} \sum_{j=0}^{n-1} B_{i j k}\left(t, \frac{\partial}{\partial x}, \frac{\partial}{\partial t}\right) u\right|_{x=a_{i}(t)}=h_{k}(t), \quad k=1, \ldots, N, \quad t \in[0, T] .
$$

Here $B_{i j k}\left(t, \frac{\partial}{\partial x}, \frac{\partial}{\partial t}\right)$ is a linear homogeneous differential operator of order $j$ such that its coefficients are continuous functions of the variable $t ; g_{i}(x)$ and $h_{k}(t)$ are given functions; $N=\operatorname{card} I_{1}^{+}+\operatorname{card} I_{2}^{-}$.

Suppose $g_{i}(x) \in C^{n-i-1}\left[a_{1}, a_{2}\right], i=0,1, \ldots, n-1, h_{k}(t) \in C[0, T], k=1, \ldots, N$. Equations (6) show that the operator $B_{i j k}$ must be a string of length $m$

$$
B_{i j k}\left(t, \frac{\partial}{\partial x}, \frac{\partial}{\partial t}\right)=\left(B_{i j k}^{1}\left(t, \frac{\partial}{\partial x}, \frac{\partial}{\partial t}\right), \ldots, B_{i j k}^{m}\left(t, \frac{\partial}{\partial x}, \frac{\partial}{\partial t}\right)\right)
$$

with elements being scalar homogeneous differential operators

$$
B_{i j k}^{p}\left(t, \frac{\partial}{\partial x}, \frac{\partial}{\partial t}\right)=\sum_{q=0}^{j} B_{i j k}^{p q}(t) \frac{\partial^{j}}{\partial x^{q} \partial t^{j-q}}, \quad p=1, \ldots, m
$$

of order $j$.

By $P_{i j}^{r s}(x, t), r, s=1, \ldots, m$, denote the elements of the matrix $P_{i j}(x, t)$. Put

$$
\alpha_{k,(j-1) m+s}^{1}(t)=\sum_{r=1}^{m} \sum_{q=0}^{n-1} B_{1, n-1, k}^{r q}(t) P_{q+1, j}^{r s}\left(a_{1}(t), t\right), \quad k=1, \ldots, N, \quad(j-1) m+s \in I_{1}^{+} ;
$$




$$
\alpha_{k,(j-1) m+s}^{2}(t)=\sum_{r=1}^{m} \sum_{q=0}^{n-1} B_{2, n-1, k}^{r q}(t) P_{q+1, j}^{r s}\left(a_{2}(t), t\right), \quad k=1, \ldots, N, \quad(j-1) m+s \in I_{2}^{-} .
$$

Let $\nu$ and $\mu$ be the cardinalities of the sets $I_{1}^{+}$and $I_{2}^{-}$, respectively. Suppose $\alpha_{1}(t)$ is the square matrix of order $N$ such that its first $\nu$ columns are formed from $\alpha_{k,(j-1) m+s}^{1}(t)$, and the rest $\mu$ ones are formed from $\alpha_{k,(j-1) m+s}^{2}(t)$. The index $k$ corresponds to the line number. In every column the index $k$ runs through the values $1, \ldots, N$ in the same order. We assume that the condition

$$
\left|\alpha_{1}(t)\right| \equiv \operatorname{det} \alpha_{1}(t) \neq 0, \quad \text { for all } t \in[0, T]
$$

holds. It is an analogue of the well-known Lopatinsky condition for the case of problem (1), (5), (6). In what follows, we will call (7) the solvability condition of the problem. If $\nu+\mu=0$, then boundary conditions (6) are missing and the condition (7) is not required. In this case, we have the Cauchy problem for system (1) with initial conditions (5).

Let us denote

$$
\begin{gathered}
\|u\|_{r, G}^{2}=\iint_{G} \sum_{i=1}^{m} \sum_{j=0}^{r} \sum_{k=0}^{j}\left(\frac{\partial^{j} u_{i}}{\partial t^{j-k} \partial x^{k}}\right)^{2} d x d t ; \quad\|f\|^{2}=\iint_{G} \sum_{i=1}^{m} f_{i}^{2} d x d t ; \\
\|g\|^{2}=\int_{a_{1}}^{a_{2}} \sum_{i=0}^{n-1} \sum_{j=1}^{m}\left(\frac{d^{n-i-1} g_{i j}}{d x^{n-i-1}}\right)^{2} d x ; \quad\|h\|^{2}=\int_{0}^{T} \sum_{k=0}^{N} h_{k}^{2} d t,
\end{gathered}
$$

where $g_{i 1}, \ldots, g_{i m}$ are the elements of the column $g_{i}$.

Theorem 1. Under all the above conditions, for any $C^{n-1}(G)$-solution $u=u(x, t)$ to problem (1), (5), (6) the estimate

$$
\|u\|_{n-1, G}^{2} \leqslant C\left(\|f\|^{2}+\|g\|^{2}+\|h\|^{2}\right)
$$

holds. A constant $C>0$ depends only on the coefficients of system (1), on the ones of boundary conditions (6), and on the domain $G$ measure.

Proof. Put

$$
V_{i}(x, t) \equiv M_{i}\left(x, t, \frac{\partial}{\partial x}, \frac{\partial}{\partial t}\right) u, \quad i=1, \ldots, n .
$$

Let $v_{(i-1) m+1}, \ldots, v_{i m}$ be components of the vector $V_{i}$. Then, by (3), (4), system (1) can be written in one of $n$ equivalent forms

$$
\frac{\partial V_{i}}{\partial t}+\Lambda_{i} \frac{\partial V_{i}}{\partial x}+\sum_{j=1}^{n} a_{i j} V_{j}=R_{i}\left(x, t, \frac{\partial}{\partial x}, \frac{\partial}{\partial t}\right) u+P^{i 1} f, \quad i=1, \ldots, n,
$$

where $a_{i j}(x, t)$ are known square matrices of order $m ; R_{j}$ are linear combinations of matrix coefficients at derivatives up to order $n-2$ inclusive; the elements of this matrices are continuous on $\bar{G}$.

Initial conditions (5) are reduced to

$$
V_{i}(x, 0)=\varphi_{i}(x) \equiv \sum_{j=1}^{n} P^{i j}(x, 0) \frac{d^{j-1} g_{n-j}(x)}{d x^{j-1}}, \quad i=1, \ldots, n .
$$


Due to (2), (3), (10), boundary conditions (6) can be written as

$$
\begin{aligned}
& \sum_{i=1}^{2} \sum_{j=1}^{n} \sum_{q=0}^{n-1} \sum_{r, s=1}^{m} B_{i, n-1, k}^{r q}(t) P_{q+1, j}^{r s}\left(a_{i}(t), t\right) v_{(j-1) m+s}\left(a_{i}(t), t\right)= \\
& =h_{k}(t)-\left.\sum_{i=1}^{2} \sum_{j=0}^{n-2} B_{i j k}\left(t, \frac{\partial}{\partial x}, \frac{\partial}{\partial t}\right) u\right|_{x=a_{i}(t)}, \quad k=1, \ldots, N .
\end{aligned}
$$

Suppose that (7) holds, then the above equalities can be written equivalently as

$$
\begin{gathered}
v_{i}\left(a_{1}(t), t\right)=\sum_{j \in I_{1}^{-}} \sigma_{i j}^{1}(t) v_{j}\left(a_{1}(t), t\right)+\sum_{j \in I_{2}^{+}} \sigma_{i j}^{2}(t) v_{j}\left(a_{2}(t), t\right)+ \\
+\sum_{k=1}^{N} \sigma_{i k}(t)\left\{h_{k}(t)-\left.\sum_{r=1}^{2} \sum_{j=0}^{n-2} B_{i j k}\left(t, \frac{\partial}{\partial x}, \frac{\partial}{\partial t}\right) u\right|_{x=a_{r}(t)}\right\}, \quad i \in I_{1}^{+}, \\
v_{i}\left(a_{2}(t), t\right)=\sum_{j \in I_{1}^{-}} \rho_{i j}^{1}(t) v_{j}\left(a_{1}(t), t\right)+\sum_{j \in I_{2}^{+}} \rho_{i j}^{2}(t) v_{j}\left(a_{2}(t), t\right)+ \\
+\sum_{k=1}^{N} \rho_{i k}(t)\left\{h_{k}(t)-\left.\sum_{r=1}^{2} \sum_{j=0}^{n-2} B_{i j k}\left(t, \frac{\partial}{\partial x}, \frac{\partial}{\partial t}\right) u\right|_{x=a_{r}(t)}\right\}, \quad i \in I_{2}^{-},
\end{gathered}
$$

where $\sigma_{i j}^{1}(t), \sigma_{i k}^{2}(t), \sigma_{i j}(t) \rho_{i j}^{1}(t), \rho_{i j}^{2}(t), \rho_{i j}(t)$ are known continuous functions on $[0, T]$.

Let $\gamma>0$ be some constant and $Q_{1}(x, t), \ldots, Q_{n m}(x, t)$ positive continuously differentiable functions on $\bar{G}$. We denote $V_{i}^{*}=\operatorname{diag}\left(v_{(i-1) m+1}, \ldots, v_{i m}\right), Q_{i}^{*}=\left(Q_{(i-1) m+1}, \ldots, Q_{i m}\right)$. Multiply left-hand side of $(11)$ by $2 e^{-\gamma t} Q_{i}^{*}(x, t) V_{i}^{*}(x, t)$. Then sum up obtained equalities over $i$ from 1 to $n$ and integrate the result over subset $G_{\tau} \subset G$, where $0<\tau \leq T$. After simple transformations, we get

$$
\iint_{G_{\tau}} e^{-\gamma t} F(v) d x d t+\int_{0}^{\tau} e^{-\gamma t} S(v) d t=N_{1}+N_{2}+N_{3}-N_{4},
$$

where

$$
\begin{gathered}
N_{1}=\iint_{G_{\tau}} 2 e^{-\gamma t} \sum_{i=1}^{n} Q_{i}^{*}(x, t) V_{i}^{*}(x, t) R_{i}\left(x, t, \frac{\partial}{\partial x}, \frac{\partial}{\partial t}\right) u d x d t \\
N_{2}=\iint_{G_{\tau}} 2 e^{-\gamma t} \sum_{i=1}^{n} Q_{i}(x, t) V_{i}^{*}(x, t) P^{i 1}(x, t) f(x, t) d x d t \\
N_{3}=\int_{a_{1}} \sum_{i=1}^{a_{2}} Q_{i}(x, 0) v_{i}^{2}(x, 0) d x, \quad N_{4}=\int_{a_{1}(\tau)}^{a_{2}(\tau)} e^{-\gamma \tau} \sum_{i=1}^{n m} Q_{i}(x, \tau) v_{i}^{2}(x, \tau) d x \\
F(v)=\gamma \sum_{i=1}^{n m} Q_{i} v_{i}^{2}+2 \sum_{i, j=1}^{n} Q_{i}^{*} V_{i}^{*} a_{i j} V_{j}-\sum_{i=1}^{n}\left(\frac{\partial Q_{i}^{*}}{\partial t} V_{i}^{*} V_{i}+\frac{\partial Q_{i}^{*}}{\partial x} V_{i}^{*} \Lambda_{i} V_{i}+\right. \\
\left.+Q_{i}^{*} V_{i}^{*} \frac{\partial \Lambda_{i}}{\partial x} V_{i}\right) \equiv \gamma \sum_{i=1}^{n m} Q_{i} v_{i}^{2}+\sum_{i, j=1}^{n m} b_{i j} v_{i} v_{j}
\end{gathered}
$$




$$
S(v)=\sum_{i=1}^{n m}\left(Q_{i}\left(a_{2}(t), t\right) w_{i}^{2}(t) v_{i}^{2}\left(a_{2}(t), t\right)-Q_{i}\left(a_{1}(t), t\right) w_{i}^{1}(t) v_{i}^{2}\left(a_{1}(t), t\right)\right) \equiv S_{2}(v)-S_{1}(v) .
$$

Obviously, $b_{i j}(x, t)$ are continuous functions on $\bar{G}$.

Given a set of positive continuous functions $Q_{i}(x, t)$ on $\bar{G}$, we choose a number $\gamma$ so large that the quadratic form $F$ is positive-definite one

$$
F(v) \geqslant C_{1} \sum_{i=1}^{n m} v_{i}^{2}(x, t), \quad C_{1}>0 .
$$

Let us write $S_{2}(v)=S_{2}^{+}(v)+S_{2}^{-}(v)$, where addends of the sum $S_{2}$ are indexed by $i \in I_{2}^{+}$, and ones of $S_{2}^{-}$does so by $i \in I_{2}^{-}$. Analogously, $S_{1}(v)=S_{1}^{+}(v)+S_{1}^{-}(v)$, where $S_{1}^{+}$and $S_{1}^{-}$ are corresponded to the indices $i \in I_{1}^{+}$and $i \in I_{1}^{-}$, respectively.

Since $w_{i}^{2}(t)>0, i \in I_{2}^{+}$and $w_{i}^{1}(t)<0, i \in I_{1}^{-}$, for $t \in[0, T]$, then for arbitrary strictly positive $Q_{i}\left(a_{1}(t), t\right), i \in I_{1}^{-}$, and $Q_{i}\left(a_{2}(t), t\right), i \in I_{2}^{+}$, the difference $S_{2}^{+}(v)-S_{1}^{-}(v)$ is a positive-definite quadratic form of $v_{i}\left(a_{1}(t), t\right), i \in I_{1}^{-}$, and $v_{i}\left(a_{2}(t), t\right), i \in I_{2}^{+}$. In the sums $S_{1}^{+}$and $S_{2}^{-}$, let us replace $v_{i}\left(a_{1}(t), t\right), i \in I_{1}^{+}$, and $v_{i}\left(a_{2}(t), t\right), i \in I_{2}^{-}$by their expressions (13).

Let $M, m_{1}, m_{2}$ be positive integers. Consider as $Q_{i}(x, t), i=1, \ldots, m n$, arbitrary smooth functions on $\bar{G}$ satisfying the conditions

$$
\begin{aligned}
& \left|Q_{i}\left(a_{1}(t), t\right) w_{i}^{1}(t)\right| \geqslant M, \quad i \in I_{1}^{-} ; \\
\left|Q_{i}\left(a_{2}(t), t\right) w_{i}^{2}(t)\right| \geqslant M, & i \in I_{2}^{+} ; \\
m_{1} \leqslant\left|Q_{i}\left(a_{1}(t), t\right) w_{i}^{1}(t)\right| \leqslant m_{2}, & i \in I_{1}^{+} ; \\
m_{1} \leqslant\left|Q_{i}\left(a_{2}(t), t\right) w_{i}^{2}(t)\right| \leqslant m_{2}, & i \in I_{2}^{-} .
\end{aligned}
$$

We take $M$ so large, and $m_{1}, m_{2}$ so small that the following inequality

$$
\begin{aligned}
& S(v) \geqslant C_{2}\left(\sum_{i \in I_{1}^{-}} v_{i}^{2}\left(a_{1}(t), t\right)+\sum_{i \in I_{2}^{+}} v_{i}^{2}\left(a_{2}(t), t\right)\right)- \\
& -C_{3}\left(\left.\sum_{q=1}^{m} \sum_{r=1}^{2} \sum_{j=0}^{n-2} \sum_{s=0}^{j}\left(\frac{\partial^{j} u_{q}}{\partial t^{j-s} \partial x^{s}}\right)^{2}\right|_{x=a_{r}(t)}+\|h\|^{2}\right)
\end{aligned}
$$

holds, where $C_{2}, C_{3}$ are some positive constants.

It is readily seen that

$$
N_{4} \geqslant m_{1} e^{-\gamma \tau} \int_{a_{1}(\tau)}^{a_{2}(\tau)} \sum_{i=1}^{n m} v_{i}^{2}(x, \tau) d x, \quad\left|N_{1}+N_{2}+N_{3}\right| \leqslant C_{4}\left(\|u\|_{n-1, G_{\tau}}^{2}+\|f\|^{2}+\|g\|^{2}\right), C_{4}>0
$$

in (14). Also, it is easy to deduce that

$$
\left.\int_{0}^{\tau} \sum_{q=1}^{m} \sum_{r=1}^{2} \sum_{j=0}^{n-2} \sum_{s=0}^{j}\left(\frac{\partial^{j} u_{q}}{\partial t^{j-s} \partial x^{s}}\right)^{2}\right|_{x=a_{r}(t)} d t \leqslant C_{5}\left(\|u\|_{n-1, G_{\tau}}^{2}+\|g\|^{2}\right), \quad C_{5}>0 .
$$

Then, from what has been said and (14), we immediately obtain

$$
\int_{a_{1}(\tau)}^{a_{2}(\tau)} \sum_{i=1}^{n m} v_{i}^{2}(x, \tau) d x \leqslant C_{6}\left(\|u\|_{n-1, G_{\tau}}^{2}+\|f\|^{2}+\|g\|^{2}+\|h\|^{2}\right), \quad C_{6}>0
$$


and as a result

$$
\begin{gathered}
\int_{a_{1}(\tau)}^{a_{2}(\tau)} \sum_{q=1}^{m} \sum_{j=0}^{n-1} \sum_{k=0}^{j}\left(\frac{\partial^{j} u_{q}(x, \tau)}{\partial t^{j-k} \partial x^{k}}\right)^{2} d x \leqslant C_{7}\left(\int_{a_{1}(\tau)}^{a_{2}(\tau)} \sum_{i=1}^{n m} v_{i}^{2}(x, \tau) d x+\|u\|_{n-1, G_{\tau}}^{2}+\|g\|^{2}\right) \leqslant \\
\leqslant C_{8}\left(\|u\|_{n-1, G_{\tau}}^{2}+\|f\|^{2}+\|g\|^{2}+\|h\|^{2}\right),
\end{gathered}
$$

where a constant $C_{8}>0$ does not depend on $u$. Using Gronwall's lemma, we get (9).

4. The case of a system with piecewise smooth coefficients. The aim of this section is to establish an a priori estimate, analogous to (9), for the solution to a general mixed problem for a hyperbolic system with discontinuous coefficients. Many questions arising by consideration of such problems are described in [4].

Let $R \geqslant 1$ be a given positive integer. Let $l_{r}, r \in\{1, \ldots, R+1\}$, be a curve defined by the equation $x=a_{r}(t), t \in[0, T]$. Suppose $a_{r}(t) \in C^{1}[0, T]$. We will assume that $a_{r}(t)<a_{r+1}(t)$, $r=1, \ldots, R$, for all $t \in[0, T]$. Denote

$$
G^{r}=\left\{(x, t) \in \mathbb{R}^{2}: 0<t \leqslant T, a_{r}(t)<x<a_{r+1}(t)\right\}, \quad G=\bigcup_{r=1}^{R} G^{r} .
$$

On every $G^{r}$, let us consider a strictly hyperbolic system

$$
\sum_{i=0}^{n} A_{i}^{r}\left(x, t, \frac{\partial}{\partial x}, \frac{\partial}{\partial t}\right) u^{r}=f^{r}(x, t), \quad r=1, \ldots, R
$$

of the form (1), where $A_{i}^{r}$ is the linear homogeneous matrix differential operator

$$
A_{i}^{r}\left(x, t, \frac{\partial}{\partial x}, \frac{\partial}{\partial t}\right) u^{r}=\sum_{i=0}^{i} A_{i j}^{r}(x, t) \frac{\partial^{i} u^{r}}{\partial x^{j} \partial t^{i-j}}
$$

of order $i$. Its coefficients $A_{i j}^{r}$ are square matrices of order $m ; u^{r}$ and $f^{r}$ are columns of height $m$ with components $u_{1}^{r}, \ldots, u_{m}^{r}$ and $f_{1}^{r}, \ldots, f_{m}^{r}$, respectively. Put $A_{n 0}^{r}(x, t) \equiv E_{m}$, $r=1, \ldots, R$, just like before. Suppose $A_{n j}^{r} \in C^{1}\left(\overline{G^{r}}\right), j=1, \ldots, n ; A_{i j}^{r}(x, t), f^{r}(x, t) \in C\left(\overline{G^{r}}\right)$, $i=0, \ldots, n-1, j=0,1, \ldots, i, r=1, \ldots, R$.

For every $r \in\{1, \ldots, R\}$, denote by $-\lambda_{1}^{r}(x, t), \ldots,-\lambda_{n m}^{r}(x, t)$ the $\lambda$-roots of the characteristic equation $\operatorname{det} A_{n}^{r}(x, t, 1, \lambda)=0$. Suppose the differences $w_{i r}^{1} \equiv \lambda_{i}\left(a_{r}(t), t\right)-a_{r}^{\prime}(t)$ and $w_{i r}^{2} \equiv \lambda_{i}\left(a_{r+1}(t), t\right)-a_{r+1}^{\prime}(t)$ do not have zeros on $[0, T]$. Let $I_{1 r}^{+}$(resp. $\left.I_{1 r}^{-}\right)$denote the set of those indices $i$ for which $w_{i r}^{1}(t)>0$ (resp. $w_{i r}^{1}(t)<0$ ). Likewise, $I_{2 r}^{+}$(resp. $I_{2 r}^{-}$) does so for $w_{i}^{2 r}(t)>0$ (resp. $\left.w_{i}^{2 r}(t)<0\right)$.

For (15), we consider the initial conditions

$$
\frac{\partial^{i} u^{r}(x, 0)}{\partial t^{i}}=g_{i}^{r}(x), \quad i=0,1, \ldots, n-1, \quad r=1, \ldots, R, \quad x \in\left[a_{r}(0), a_{r+1}(0)\right],
$$

together with following boundary conditions on $l_{1}$ and $l_{R+1}$ and conjugation conditions on $l_{2}, \ldots, l_{R}$

$$
\left.\sum_{r=1}^{R} \sum_{i=r}^{r+1} \sum_{j=0}^{n-1} B_{i j k}^{r}\left(t, \frac{\partial}{\partial x}, \frac{\partial}{\partial t}\right) u^{r}\right|_{x=a_{i}(t)}=h_{k}(t), \quad k=1, \ldots, N_{1}, \quad t \in[0, T] .
$$


Here $N_{1}=\sum_{r=1}^{R}\left(\operatorname{card} I_{1 r}^{+}+\operatorname{card} I_{2 r}^{-}\right) ; B_{i j k}^{r}$ are the operators

$$
B_{i j k}^{r}=\left(B_{i j k}^{r 1}, \ldots, B_{i j k}^{r m}\right), \quad B_{i j k}^{r p} \equiv \sum_{q=0}^{j} B_{i j k}^{r p q}(t) \frac{\partial^{j}}{\partial x^{q} \partial t^{j-q}} .
$$

Their structure, for every $r$, is the same as for the operators $B_{i j k}$ in (6).

Suppose $g_{i}^{r} \in C^{n-i-1}\left[a_{r}(0), a_{r+1}(0)\right]$, and the coefficients and free terms of (17) are continuous on $[0, T]$. Evidently, at $R=1$, the formulated problem is the one from the previous section.

For every fixed $r=1, \ldots, R$, we introduce the matrices $A^{r}(x, t)$ and $P^{r}(x, t)$ as well as $A$ and $P$ in Section 2. Let $P_{i j}^{r}, i, j=1, \ldots, n$, be the block elements of the matrix $P^{r}$, and $P_{i j}^{r p s}, p, s=1, \ldots, m$, the elements of $P_{i j}^{r}$. Put

$$
\begin{gathered}
\alpha_{k,(j-1) m+s}^{r 1}(t)=\sum_{p=1}^{m} \sum_{q=0}^{n-1} B_{r, n-1, k}^{r p q}(t) P_{q+1, j}^{r p s}\left(a_{r}(t), t\right), \quad(j-1) m+s \in I_{1 r}^{+}, \\
\alpha_{k,(j-1) m+s}^{r 2}(t)=\sum_{p=1}^{m} \sum_{q=0}^{n-1} B_{r+1, n-1, k}^{r p q}(t) P_{q+1, j}^{r p s}\left(a_{r+1}(t), t\right), \quad(j-1) m+s \in I_{2 r}^{-}
\end{gathered}
$$

for all $k=1, \ldots, N_{1}$ and $r=1, \ldots, R$.

Let $\alpha_{2}(t)$ be a square matrix of order $N_{1}$ such that $\alpha_{k p}^{r q}(t)$, introduced above, are its elements. Here $p=(j-1) m+s \in I_{1 r}^{+}$for $q=1$ but $p(j-1) m+s \in I_{2 r}^{-}$for $q=2$ and $j=1, \ldots, n, s=1, \ldots, m$. For every fixed triple $p, r, q$, the elements $\alpha_{k p}^{r q}(t), k=1, \ldots, N_{1}$, present a column of the matrix.

Suppose the condition

$$
\operatorname{det} \alpha_{2}(t) \neq 0, \quad \text { for all } t \in[0, T],
$$

similar to that of Lopatinsky is satisfied. It is also called the solvability conditions of problem (15)-(17).

We denote by $\left\|u^{r}\right\|_{n, G^{r}}^{2},\left\|f^{r}\right\|_{G^{r}}^{2}$ the corresponding norms defined by (8) for $G=G^{r}$.

Theorem 2. Under the assumptions of the current section, for any $C^{n-1}(G)$-solution of problem (15)-(17) the estimate

$$
\sum_{r=1}^{R}\left\|u^{r}\right\|_{n-1, G^{r}}^{2} \leqslant C \sum_{r=1}^{R}\left(\left\|f^{r}\right\|_{G^{r}}^{2}+\left\|g^{r}\right\|^{2}\right)+C\|h\|^{2}
$$

holds. Here a constant $C>0$ does not depend on the solution.

Proof. The theorem can be proved in the same scheme as can Theorem 1, so let us restrict ourselves only to general remarks.

On every $G^{r}$, we consider the operators $M_{i}^{r}$ and the vector functions $V_{i}^{r}$ with components $v_{(i-1) m+1}^{r}, \ldots, v_{i m}^{r}, i=1, \ldots, n$, analogous to ones in (2) and (10). For functions $V_{i}^{r}$ we obtain a first-order system (11), assuming that all coefficients in (11) are marked by the upper index $r$. Respectively, initial conditions (16) induce the ones for $V_{i}^{r}$. From (17) due to (18), we can expressed $v_{i}^{r}\left(a_{r}(t), t\right), i \in I_{1 r}^{+}, r=1, \ldots, R$ and $v_{i}^{r}\left(a_{r+1}(t), t\right), i \in I_{2 r}^{-}, r=2, \ldots, R+1$, 
in terms of $h_{k}(t), k=1, \ldots, N_{1}$, the values of $u^{r}$ and of their derivatives up to order $n-2$ inclusive on the lines $l_{r}, r=1, \ldots, R+1$, and the values of the rest function $v_{i}^{r}$ on the ones.

Thus, the equalities of form (13) are obtained. Suppose $\gamma$ is sufficiently large and the same for all $G^{r}$, and the functions $Q_{1}(x, t), \ldots, Q_{n}(x, t)$ are chosen, on each component $G^{r}$ separately, as in previous section. They are piecewise smooth ones on $G$. Thus we have an equality of form (14). Finally, acting as in Section 3 with obvious changes, we get an inequality of type (19).

Remark 1. Suppose the initial data of the problems considered in Sections 2-4 are smoother; then one can get estimates for the norms $\|\cdot\|_{N, G}$ of solutions, where $N>n-1$.

Remark 2. By slightly changing the proofs of Theorems 1 and 2, one can obtain, under the appropriate conditions for the initial data, a priori estimates for $L_{p}$-norms of solutions and of their derivatives up to order of not less than $n-1$.

\section{Correct solvability of a nonlocal problem with nonseparated and integral initial}

data. Various methods have been used to prove the correct solvability of mixed problems for two-dimensional hyperbolic equations and systems with smooth coefficients. Depending on the proof technique, there are three basic ones ([16]). The first is the approximation of smooth functions by analytic ones; the second is the using of the finite difference method (both covering the multidimensionality of spatial variables); and the third is the characteristics method (specific in the case of one spatial variable). Various problems for a second order equation have been studied in [14,27,34-37,39,47]. Mixed problems for first-order systems have been considered in $[1,8,18,20,21,28,33,40-43,56,62,69-75,77,79]$. Cases of an single equation or high order system of equations have been investigated in $[2,7,10,22,24,44$, $45,47,49,51,60,61,70,71,76]$. In $[54,57,58]$ the simplest mixed problems for equations and systems with boundary conditions involving integrals, over the spatial variable, of the desired solution have been studied. Various models of problems with nonlocal conditions, such as nonseparated and integral ones, have also been studied in $[26,32,63]$.

This section deals with conditions for correct solvability of a general problem, with integral constraints, for system (1) defined in Section 2 on $G$.

We find a solution of (1) on $G$ satisfying the initial conditions (5) and constraints

$$
\begin{gathered}
\sum_{i=0}^{n-1} \sum_{j=0}^{i} \sum_{p=1}^{m}\left(\left.\sum_{q=1}^{2} B_{q i k}^{p j}(t) \frac{\partial^{i} u_{p}}{\partial t^{i-j} \partial x^{j}}\right|_{x=a_{q}(t)} ^{+}\right. \\
\left.+\int_{a_{1}(t)}^{a_{2}(t)} C_{i k}^{p j}(\xi, t) \frac{\partial^{i} u_{p}(\xi, t)}{\partial t^{i-j} \partial \xi^{j}} d \xi\right)=h_{k}(t), \quad k=1, \ldots, N_{0}, \quad t \in[0, T] ; \\
\sum_{i=0}^{n-1} \sum_{j=0}^{i} \sum_{p=1}^{m} \int_{a_{1}(t)}^{a_{2}(t)} C_{i k}^{p j}(\xi, t) \frac{\partial^{i} u_{p}(\xi, t)}{\partial t^{i-j} \partial \xi^{j}} d \xi=h_{k}(t), \quad k=N_{0}+1, \ldots, N,
\end{gathered}
$$

where $B_{q i k}^{p j}, C_{i k}^{p j}, h_{k}$ are known functions, $0 \leqslant N_{0} \leqslant N$. In extreme cases $N_{0}=0$ and $N_{0}=N$ the corresponding conditions are absent. 
Suppose initial conditions (5) and condition (20) are agreed at $\left(a_{1}, 0\right)$ and $\left(a_{2}, 0\right)$. Namely

$$
\begin{gathered}
\sum_{i=0}^{n-1} \sum_{j=0}^{i} \sum_{p=1}^{m}\left(\sum_{q=1}^{2} B_{q i k}^{p j}(0) g_{i-j, p}^{(j)}\left(a_{q}\right)+\int_{a_{1}}^{a_{2}} C_{i k}^{p j}(\xi, 0) g_{i-j, p}^{(j)}(\xi) d \xi\right)=h_{k}(0), \quad k=1, \ldots, N_{0} . \\
\sum_{i=0}^{n-1} \sum_{j=0}^{i} \sum_{p=1}^{m} \int_{a_{1}(t)}^{a_{2}(t)} C_{i k}^{p j}(\xi, 0) g_{i-j, p}^{(j)}(\xi) d \xi=h_{k}(0), \quad k=N_{0}+1, \ldots, N .
\end{gathered}
$$

Let us construct matrices $\beta_{1}(t), \beta_{2}(t), \beta_{3}(t), \beta_{4}(t)$ as follows. The matrices $\beta_{1}(t)$ and $\beta_{2}(t)$, likewise to ones in Section 4 , consist of elements $\alpha_{k,(j-1) m+s}^{1}(t)$ and $\alpha_{k,(j-1) m+s}^{2}(t)$, respectively, where $k=1, \ldots, N_{0}$. The matrices $\beta_{3}(t)$ and $\beta_{4}(t)$ are composed in the same way, but they consist of elements

$$
\alpha_{k,(j-1) m+s}^{3}(t)=\sum_{r=1}^{m} \sum_{q=0}^{n-1} C_{n-1, k}^{r q}\left(a_{1}(t), t\right) P_{q+1, j}^{r s}\left(a_{1}(t), t\right) w_{(j-1) m+s}^{1}\left(a_{1}(t), t\right),
$$

where $k=N_{0}+1, \ldots, N,(j-1) m+s \in I_{1}^{+}$, and

$$
\alpha_{k,(j-1) m+s}^{4}(t)=-\sum_{r=1}^{m} \sum_{q=0}^{n-1} C_{n-1, k}^{r q}\left(a_{2}(t), t\right) P_{q+1, j}^{r s}\left(a_{2}(t), t\right) w_{(j-1) m+s}^{2}\left(a_{2}(t), t\right),
$$

where $k=N_{0}+1, \ldots, N,(j-1) m+s \in I_{2}^{-}$.

Note for the pairs of matrices $\beta_{1}(t), \beta_{2}(t)$ and $\beta_{3}(t), \beta_{4}(t)$, the index $k$ runs in the same order over the sets $1, \ldots, N_{0}$ and $N_{0}+1, \ldots, N$, respectively. The same being true for the pairs $\beta_{1}(t), \beta_{2}(t)$ and $\beta_{3}(t), \beta_{4}(t)$, but for index $(j-1) m+s$ running over $I_{1}^{+}$and $I_{2}^{-}$.

Therefore the square matrix $\beta(t)=\left(\begin{array}{ll}\beta_{1}(t) & \beta_{2}(t) \\ \beta_{3}(t) & \beta_{4}(t)\end{array}\right)$ has order $N$.

Let the solvability condition

$$
\operatorname{det} \beta(t) \neq 0 \text { for all } t \in[0, T]
$$

be satisfied. It is analogous, for given problem, to the Lopatinsky condition.

Theorem 3. Suppose the following conditions hold:

1 ) in (1), the coefficients of the operator $A_{n}$ are continuously differentiable; the coefficients rest of $A_{i}, i<n$, and the free term $f$ are continuous on $\bar{G}$;

2) the initial functions $g_{i}$ are continuously differentiable $n-i-1$ times on $\left[a_{1}, a_{2}\right]$;

3) for $k=1, \ldots, N_{0}$, the coefficients and free terms in (20) are continuous on $\bar{G}$ and $[0, T]$ respectively;

4) for $k=N_{0}+1, \ldots, N$, the coefficients $C_{n-1, k}^{p j}(x, t)$ are continuously differentiable in $x$ and $t$, and the coefficients $C_{i k}^{p j}(x, t), i<n-1$, are continuous and continuously differentiable in $t$ on $\bar{G}$;

$5)$ the functions $a_{1}(t), a_{2}(t), h_{k}(t), k=N_{0}+1, \ldots, N$, are continuously differentiable on $[0, T]$; 
6) agreement conditions (21) and solvability conditions (22) are satisfied.

Then there exists a unique solution of class $C^{n-1}(\bar{G})$ to problem (1), (5), (20) continuously depending on the right parts of (1) and (20), and initial functions (5) in the sense of metrics (8).

The meaning of this solution will be specified below.

Proof. The proof is based on the method of characteristics and is to reduce the problem to an equivalent system of Volterra integral equations. This system is solved by the iteration method.

Introducing functions (10), let us write equation (1) in form (11), namely as

$$
\frac{\partial v_{i}}{\partial t}+\lambda_{i}(x, t) \frac{\partial v_{i}}{\partial x}=F_{i}(x, t, u, v), \quad i=1, \ldots, n m
$$

Here $F_{i}$ is a linear function with continuous coefficients on $\bar{G}$; the ones are depended on $v_{i}$, $i=1, \ldots, n m$, on $u_{j}, j=1, \ldots, m$, and on all derivatives of the functions $u_{j}$ up to order $n-2$ inclusive; $v$ is a vector with components $v_{1}, \ldots, v_{n m}$. Taking into account (12), initial conditions (5) can be written as

$$
v_{i}(x, 0)=\psi_{i}(x), \quad i=1, \ldots, n m, \quad x \in\left[a_{1}, a_{2}\right] .
$$

Let us consider the auxiliary functions

$$
\begin{array}{ll}
v_{i}\left(a_{1}(t), t\right)=\nu_{i}^{1}(t), & i \in I_{1}^{+}, \\
v_{i}\left(a_{2}(t), t\right)=\nu_{i}^{2}(t), & i \in I_{2}^{-} .
\end{array}
$$

Suppose $x=\varphi_{i}(t, \xi, \tau)$ is a solution of the characteristic equation $x_{t}^{\prime}=\lambda_{i}(x, t)$ satisfying the initial condition $x(\tau)=\xi$, where $(\xi, \tau) \in \bar{G}$. Let $L_{i}(\xi, \tau)$ be the corresponding characteristic passing through $(\xi, \tau)$, let it be extended backwards in time to the intersection with the boundary of $G$, and $t_{i}(\xi, \tau)$ the smallest value of $t$ for its points. Evidently, $0 \leqslant t_{i}(\xi, \tau) \leqslant \tau$. If $t_{i}(\xi, \tau)>0$, then $\varphi_{i}\left(t_{i}(\xi, \tau), \xi, \tau\right)$ is equal to $a_{1}\left(t_{i}(\xi, \tau)\right)$ or to $a_{2}\left(t_{i}(\xi, \tau)\right)$.

Accordingly, the domain $G$ is split into three parts

$$
\begin{array}{ll}
G_{i}^{0}:=\left\{t_{i}(\xi, \tau) \equiv 0\right\}, & G_{i}^{1}:=\left\{t_{i}(\xi, \tau)>0, \varphi_{i}\left(t_{i}(\xi, \tau), \xi, \tau\right) \equiv a_{1}\left(t_{i}(\xi, \tau)\right)\right\}, \\
& G_{i}^{2}:=\left\{t_{i}(\xi, \tau)>0, \varphi_{i}\left(t_{i}(\xi, \tau), \xi, \tau\right) \equiv a_{2}\left(t_{i}(\xi, \tau)\right)\right\} .
\end{array}
$$

Any of the sets $G_{i}^{1}$ or $G_{i}^{2}$ may be empty.

Integrating (23) along characteristics [1, 16,46], and taking into account (24) and (25), we obtain in the domain $\bar{G}$ the system of integro-differential relationships

$$
\left.v_{i}(x, t)=w_{i}(x, t)+\int_{t_{i}(x, t)}^{t} F_{i}\left(\varphi_{i}(\tau, x, t), \tau, u\left(\varphi_{i}(\tau, x, t), \tau\right)\right), v\left(\varphi_{i}(\tau, x, t), \tau\right)\right) d \tau
$$

where

$$
w_{i}(x, t)=\left\{\begin{array}{ll}
\psi_{i}\left(\varphi_{i}(0, x, t)\right), & \text { if }(x, t) \in G_{i}^{0}, \\
\nu_{i}^{1}\left(t_{i}(x, t)\right), & \text { if }(x, t) \in G_{i}^{1}, \\
\nu_{i}^{2}\left(t_{i}(x, t)\right), & \text { if }(x, t) \in G_{i}^{2}
\end{array} \quad \text { for } \quad i=1, \ldots, n m\right.
$$


For the purpose of the function $v_{i}(x, t)$ to be continuous when passing from $G_{i}^{0}$ to $G_{i}^{1}$ and to $G_{i}^{2}$, the fulfillment of the agreement conditions

$$
\nu_{i}^{1}(0)=\psi_{i}\left(a_{1}\right), \quad i \in I_{1}^{+} ; \quad \nu_{i}^{2}(0)=\psi_{i}\left(a_{2}\right), \quad i \in I_{2}^{-}
$$

is required. Conditions (20) can be written as

$$
\begin{gathered}
\sum_{j=1}^{n} \sum_{q=0}^{n-1} \sum_{r, s=1}^{m}\left(\sum_{i=1}^{2} B_{i, n-1, k}^{r q}(t) P_{q+1, j}^{r s}\left(a_{i}(t), t\right) \nu_{(j-1) m+s}^{i}(t)+\right. \\
\left.+\int_{a_{1}(t)}^{a_{2}(t)} C_{n-1, k}^{r q}(\xi, t) P_{q+1, j}^{r s}(\xi, t) v_{(j-1) m+s}(\xi, t) d \xi\right)=H_{k}^{1}(t, u), \quad k=1, \ldots, N_{0} \\
\sum_{j=1}^{n} \sum_{q=0}^{n-1} \sum_{r, s=1}^{m} \int_{a_{1}(t)}^{a_{2}(t)} C_{n-1, k}^{r q}(\xi, t) P_{q+1, j}^{r s}(\xi, t) v_{(j-1) m+s}(\xi, t) d \xi=H_{k}^{2}(t, u), \\
k=N_{0}+1, \ldots, N,
\end{gathered}
$$

where $H_{k}^{1}$ and $H_{k}^{2}$ coincide with the free members $h_{k}(t)$ of $(20)$ minus all the left side addends containing derivatives of $u_{p}$ up to order $n-2$ inclusive.

Functions (25) must be such that for functions $v_{i}$ determined from (26), conditions (28) are satisfied. For this to be the case, in (28) we replace $v_{i}$ with right-hand sides of (26). In the one-fold integrals of the left-hand sides of the obtained equations, we do the change of variable $\xi$ by $\tau=t_{(j-1) m+s}(\xi, t)$. Then we differentiate equalities corresponding to the indices $k=N_{0}+1, \ldots, N$ with respect to $t$. As a result, from the expressions $\partial H_{k}^{2}(t, u) / \partial t$, $(n-1)$-th derivatives of the functions $u_{p}$ are appeared. We replace them with $v_{i}$ according to (3). Then we do so with regard to $v_{i}$ and $(26)$.

Finally, equalities (28) take the form

$$
\begin{aligned}
& \sum_{(j-1) m+s \in I_{1}^{+}} \alpha_{k,(j-1) m+s}^{q} \nu_{(j-1) m+s}^{q}(t)+\sum_{(j-1) m+s \in I_{2}^{-}} \alpha_{k,(j-1) m+s}^{q} \nu_{(j-1) m+s}^{q}(t)= \\
& =\int_{0}^{t} \sum_{(j-1) m+s \in I_{1}^{+}} R_{k,(j-1) m+s}^{q 1}(t, \tau) \nu_{(j-1) m+s}^{1}(\tau) d \tau+ \\
& +\int_{0}^{t} \sum_{(j-1) m+s \in I_{2}^{-}} R_{k,(j-1) m+s}^{q 2}(t, \tau) \nu_{(j-1) m+s}^{2}(\tau) d \tau+H_{k}^{q+2}(t, u, v),
\end{aligned}
$$

where $q=1$ for $k=1, \ldots, N_{0}$, and $q=2$ for $k=N_{0}+1, \ldots, N$.

Here $R_{k p}^{q 1}(t, \tau), R_{k p}^{q 2}(t, \tau)$ are known continuous functions; $H_{k}^{3}(t, u, v)$ and $H_{k}^{4}(t, u, v)$ are expressions containing $v_{i}, u_{p}$ and derivatives of $u_{p}$ up to order $n-2$ inclusive.

Taking into account (22), these equalities can be solved with respect to $\nu_{i}^{1}$ and $\nu_{i}^{2}$. Let us write the result in the matrix form

$$
\nu(t)=\int_{0}^{t} R(t, \tau) \nu(\tau) d \tau+H(t, u, v) .
$$


Here $\nu(\tau)$ is a column of height $N$ with the components $\nu_{i}^{1}$ and $\nu_{i}^{2}, R(t, \tau)$ is a known continuous kernel, $H(t, u, v)$ is a vector with components of the form $H_{k}^{q+2}(t, u, v)$.

Let $S(t, \tau)$ be resolvent of the kernel $R(t, \tau)$. Therefore the last equality can be written as

$$
\nu(t)=H(t, u, v)+\int_{0}^{t} S(t, \tau) H(\tau, u, v) d \tau .
$$

Suppose conditions (21) are held; then it is easy to show that the functions $\nu_{i}^{1}$ and $\nu_{i}^{2}$, defined by (29), satisfy condition (27) for arbitrary $u, v$.

Substituting $\nu_{i}^{1}$ and $\nu_{i}^{2}$ mentioned above in (26), we get

$$
v_{i}(x, t)=W_{i}(x, t)+U_{i 1}(x, t, u)+W_{i 1}(x, t, u, v), \quad i=1, \ldots, m n,
$$

where $W_{i}$ are known continuous functions; $U_{i 1}$ is a linear combination, with continuous coefficients, of the values of the functions $u_{p}$ and their derivatives up to order $n-2$ on the lines $l_{1}$ and $l_{2} ; W_{i 1}$ are Volterra type addends, with continuous kernels, containing $v_{i}$ and all derivatives of $u_{p}$ up to order $n-2$.

Let $L: x=a(t)$ be an arbitrary smooth curve connecting a point $(x, t) \in \bar{G}$ and the segment $\left[a_{1}, a_{2}\right]$ of the axis $O x$, and completely lying in $\bar{G}$. Denote by $D_{t} f$ the full derivative of $f$ along $L$ with respect to $t$.

Replacing $v_{i}$ in the right-hand sides of (30) with (10) and using (3), we rewrite (30) as

$$
\frac{\partial^{n-1} u}{\partial t^{n-i-1} \partial x^{i}}=T_{i}^{n-1}(x, t)+R_{i}^{n-1}(x, t, u)+S_{i}^{n-1}(x, t, u),
$$

where $T_{i}^{n-1}, R_{i}^{n-1}, S_{i}^{n-1}$ are columns of height $m$ with an obvious structure of their components.

It follows from (3) and (10) that

$$
D_{t} \frac{\partial^{n-2} u}{\partial t^{n-i-2} \partial x^{i}}=\sum_{j=1}^{n}\left(P_{i+1, j}+a^{\prime}(t) P_{i+2, j}\right) V_{j}, \quad i=0,1, \ldots, n-2 .
$$

Let us replace $V_{i}$ in the right-hand sides with (10), then replace the derivatives of $u$ of order $n-1$ with (31). Integrating the resulting equality along $L$ with respect to $t$, we get

$$
\frac{\partial^{n-2} u}{\partial t^{n-i-2} \partial x^{i}}=T_{i}^{n-2}(x, t)+R_{i}^{n-2}(x, t, u),
$$

where $T_{i}^{n-2}$ are known continuous functions, $R_{i}^{n-2}$ are Volterra type addends.

After that, we consistently form expressions

$$
\frac{\partial^{n-3} u}{\partial t^{n-i-3} \partial x^{i}}, \quad i=0,1, \ldots, n-3, \quad \frac{\partial^{n-4} u}{\partial t^{n-i-4} \partial x^{i}}, \quad i=0,1, \ldots, n-4, \text { etc. }
$$

Integrating the ones along $L$ with respect to $t$, we get relationships, similar to (32), for derivatives of order $n-3, n-4, \ldots, 0$. Finally, we substitute these derivatives up to order $n-2$ inclusive into (31), namely in $R_{i}^{n-1}$.

Then for $u$ and its all derivatives up to order $n-1$ inclusive, we obtain a system of $(n+1) n / 2$ vector Volterra-type integral equations of the form

$$
\frac{\partial^{n-i} u}{\partial t^{n-i-j} \partial x^{j}}=T_{j}^{n-i}(x, t)+R_{j}^{n-i}(x, t, u), \quad i=1, \ldots, n, \quad j=0,1, \ldots, n-i,
$$


where $T_{j}^{n-i}$ are known functions, $R_{j}^{n-i}$ are Volterra operators of $u$ and of its all derivatives up to order $n-1$ inclusive.

Under the conditions of Theorem 3, it is easy to show that there exists a unique continuous solution of system (33) on $\bar{G}$, obtaining by the iteration method. We call it the generalized solution to problem $(1),(5),(20)$ of class $C^{n-1}(\bar{G})$. The continuous dependence of the solution on the free member $f$ and on the initial functions $h_{k}$ also follows easily from (33).

Remark 3. Increasing the smoothness of the initial data, one can similarly proof the existence and uniqueness of a classical solution to the problem.

Remark 4. If $l_{1}$ and $l_{2}$ (or one of them) are characteristics of (1), then the number of conditions (20) is decreased by two (or one) units.

6. Correct solvability of a nonlocal problem with piecewise smooth initial data. A number of papers are devoted to the problem of the correct solvability of mixed problems for hyperbolic equations and systems with discontinuous coefficients on a line. In particular, in [46] a common mixed problem for a first-order system with discontinuous data has been investigated by the method of characteristics. Mixed problems with discontinuous data for a second-order equation have been studied by various methods in $[2,9,19,48,66]$. The case of one arbitrary order equation has been considered in $[48,50]$. Using the contour integral method, in $[55,64]$ a broad class of problems of special kind for higher order systems has been researched.

This section deals with the general case of a nonlocal mixed problem for a hyperbolic arbitrary order system with discontinuous data.

Under the conditions of Section 4, let us consider system (15) with initial conditions (16) and the conditions

$$
\begin{gathered}
\sum_{i=0}^{n-1} \sum_{j=0}^{i} \sum_{p=1}^{m} \sum_{r=1}^{R}\left(\left.\sum_{q=r}^{r+1} B_{q i k}^{r p j}(t) \frac{\partial^{i} u_{p}^{r}}{\partial t^{i-j} \partial x^{j}}\right|_{x=a_{q}(t)}+\right. \\
\left.+\int_{a_{r}(t)}^{a_{r+1}(t)} C_{i k}^{r p j}(\xi, t) \frac{\partial^{i} u_{p}^{r}(\xi, t)}{\partial t^{i-j} \partial \xi^{j}} d \xi\right)=h_{k}(t), \quad k=1, \ldots, K ; \\
\sum_{i=0}^{n-1} \sum_{j=0}^{i} \sum_{p=1}^{m} \sum_{r=1}^{R} \int_{a_{r}(t)}^{a_{r+1}(t)} C_{i k}^{r p j}(\xi, t) \frac{\partial^{i} u_{p}^{r}(\xi, t)}{\partial t^{i-j} \partial \xi^{j}} d \xi=h_{k}(t), \quad k=K+1, \ldots, N_{1},
\end{gathered}
$$

where $B_{q i k}^{r p j}, C_{i k}^{r p j}, h_{k}(t)$ are given functions, $0 \leqslant K \leqslant N_{1}$. This conditions are stated instead of boundary ones on $l_{1}$ and $l_{R+1}$, and conjugation conditions on $l_{2}, \ldots, l_{R}$.

It is assumed that the following agreement conditions of (16) and (34) at the points $\left(a_{r}(0), 0\right), r=1, \ldots, R+1$, are satisfied

$$
\begin{gathered}
\sum_{i=0}^{n-1} \sum_{j=0}^{i} \sum_{p=1}^{m} \sum_{r=1}^{R}\left(\left.\sum_{q=r}^{r+1} B_{q i k}^{r p j}(0) \frac{d^{j} g_{i-j, p}^{r}(x)}{d x^{j}}\right|_{x=a_{q}(0)}+\right. \\
\left.+\int_{a_{r}(0)}^{a_{r+1}(0)} C_{i k}^{r p j}(\xi, 0) \frac{d^{j} g_{i-j, p}^{r}(\xi)}{d x^{j}} d \xi\right)=h_{k}(0), \quad k=1, \ldots, K .
\end{gathered}
$$




$$
\sum_{i=0}^{n-1} \sum_{j=0}^{i} \sum_{p=1}^{m} \sum_{r=1}^{R} \int_{a_{r}(0)}^{a_{r+1}(0)} C_{i k}^{r p j}(\xi, 0) \frac{d^{j} g_{i-j, p}^{r}(\xi)}{d x^{j}} d \xi=h_{k}(0), \quad k=K+1, \ldots, N_{1}
$$

Let $\alpha_{k,(j-1) m+s)}^{r p}(t)$ be the functions introduced in Section 5 , where $k=1, \ldots, K$. For fixed $r, p, j, s$, of these quantities we make up a column $\gamma_{k,(j-1) m+s)}^{r p}(t)$ of height $K$. Then for fixed $r=1, \ldots, m$ and $p=1,2$, of these columns we do the matrices $\gamma^{r 1}(t)$ and $\gamma^{r 2}(t)$ with $(j-1) m+s \in I_{1 q}^{+}$and $(j-1) m+s \in I_{2 q}^{-}$, respectively.

Let us consider the functions

$$
\begin{gathered}
\alpha_{k,(j-1) m+s}^{r 3}(t)=\sum_{p=1}^{m} \sum_{q=0}^{n-1} C_{n-1, k}^{r p q}\left(a_{r}(t), t\right) P_{q+1, j}^{r p s}\left(a_{r}(t), t\right) w_{(j-1) m+s}^{1}\left(a_{r}(t), t\right), \\
k=K+1, \ldots, N_{1}, \quad(j-1) m+s \in I_{1 q}^{+}, \quad r=1, \ldots, R ; \\
\alpha_{k,(j-1) m+s}^{r 4}(t)=-\sum_{p=1}^{m} \sum_{q=0}^{n-1} C_{n-1, k}^{r p q}\left(a_{r+1}(t), t\right) P_{q+1, j}^{r p s}\left(a_{r+1}(t), t\right) w_{(j-1) m+s}^{2}\left(a_{r+1}(t), t\right), \\
k=K+1, \ldots, N_{1}, \quad(j-1) m+s \in I_{2 q}^{-}, \quad r=1, \ldots, R .
\end{gathered}
$$

By the same way as $\gamma^{r 1}(t)$ and $\gamma^{r 2}(t)$, of these quantities we make up the matrices $\gamma^{r 3}(t)$ and $\gamma^{r 4}(t)$ of height $N_{1}-K$.

It is assumed that

$$
\operatorname{det} \gamma(t)=\operatorname{det}\left(\begin{array}{llllll}
\gamma^{11}(t) & \cdots & \gamma^{R 1}(t) & \gamma^{12}(t) & \cdots & \gamma^{R 2}(t) \\
\gamma^{13}(t) & \cdots & \gamma^{R 3}(t) & \gamma^{14}(t) & \cdots & \gamma^{R 4}(t)
\end{array}\right) \neq 0, \quad t \in[0, T] .
$$

For the case under consideration, this condition is analogous to the Lopatinsky one. We will call it the solvability condition to problem (15), (16), (34).

Theorem 4. Suppose the following conditions hold:

1) the coefficients of the operators $A_{n}^{r}, r=1, \ldots, R$, are of class $C^{1}\left(\overline{G^{r}}\right)$, and the coefficients the rest of operators and free members $f^{r}$ of system (15) are of class $C\left(\overline{G^{r}}\right)$;

2) the initial functions $g_{i}^{r}$ are of class $C^{n-i-1}\left[a_{r}(0), a_{r+1}(0)\right], i=0,1, \ldots, n-1, \quad r=$ $1, \ldots, R$;

3) for $k=1, \ldots, K$, the coefficients $C_{i k}^{r p j}$ are of class $C(\bar{G})$, and $B_{q i k}^{r p j}$ and $h_{k}(t)$ are of class $C[0, T] ;$ for $k=K+1, \ldots, N$, the functions $C_{i k}^{r p j}(\xi, t)$ and $h_{k}(t)$ are of class $C^{1}\left(\overline{G^{r}}\right)$ and $C^{1}[0, T]$, respectively;

4) agreement conditions (35) and solvability conditions (36) are satisfied.

Then there exists a unique $C^{n-1}$-solution to problem (15), (16), (34) on every $G_{r}$. It is continuously depended on $f^{r}(x, t), h_{k}(t)$ and $g_{i}^{r}(t)$.

Proof. The proof is similar in spirit to the one of Theorem 3, so let us restrict ourselves to some general instructions.

On every $G^{r}$, system (15) can be written as (23). Taking into account (36), the equality (34) can be solved with respect to $v_{i}^{r}\left(a_{r}(t), t\right), i \in I_{1 r}^{+}$and $v_{i}^{r}\left(a_{r+1}(t), t\right), i \in I_{2 r}^{-}$, where $r=1, \ldots, R$. 
Then, for every $r=1, \ldots, R$, we repeat the reasoning of Section 5 and use the considerations of [46] to transform the obtained system of integro-differential equations into a system of Volterra-type integral equations. As a result, we come to a system of $(n+1) n R / 2$ Volterra-type equations of the second kind with respect to $u_{i}^{r}(x, t)$ and their derivatives up to order $n-1$ inclusive. It is equivalent to the original problem. Solving this system by the method of successive approximations, we obtain the desired result.

Remark 5. Let boundaries of $\bar{G}$ be unknown, that is, we are dealing with a hyperbolic Stefan problem [59]. In this case conditions for unknown boundaries should be added, for all $t \in[0, T]$, to the initial and boundary conditions of problem (1), (5), (20). For example,

$$
\begin{gathered}
\left.a_{l}^{\prime \prime}(t)=\Phi_{l}\left(t, a_{1}(t), a_{2}(t), a_{1}^{\prime}(t), a_{2}^{\prime}(t), u\left(a_{1}(t), t\right), u\left(a_{2}(t), t\right)\right)\right), \\
a_{l}(0)=a_{l}^{0}, \quad a_{l}^{\prime}(0)=a_{l}^{1}, \quad l=1,2, \\
\left.\left.\max _{i \in I_{l}^{-}} \lambda_{i}\left(a_{l}(t), t\right)\right)<a_{l}^{\prime}(t)<\max _{i \in I_{l}^{+}} \lambda_{i}\left(a_{l}(t), t\right)\right) .
\end{gathered}
$$

Nonlinear problems (1), (5), (20), (37), and various problems with unknown boundaries for hyperbolic equations and systems have been investigated in $[3,5,6,31,32]$.

Remark 6. For the reasonig of this section, similar to that of Sections 4 and 5, it is important that no pair of lines $l_{r}$ have points in common. The intersection of some of these lines is much more difficult $[5,6,29-32,52,53]$. In $\bar{G}$, the lines of initial conditions can be degenerated to a point (the Darboux problems). Such problems have been investigated in [52,53]; and the case of the Darboux problem with unknown boundaries of $\bar{G}$ have been considered in [6,32].

7. About requirement of additional conditions. In each of the problems discussed above, it was assumed that some conditions, called the solvability conditions, are satisfied (conditions (7), (18), (22), (36)). And if the smoothness and agreement conditions of data are completely natural, then the occurrences of the solvability conditions may seem artificial at first glance. Let us show by examples that these conditions are significant.

By $G$ denote the rectangle $\left\{(x, t) \in \mathbb{R}^{2}: 0<x<1, \quad 0<t<0,5\right\}$. Let us consider the equation

$$
\frac{\partial^{2} u}{\partial t^{2}}-\frac{\partial^{2} u}{\partial x^{2}}=0
$$

on $G$. We are seeking for its solution that the initial conditions

$$
u(x, 0)=0, \quad u_{t}^{\prime}(x, 0)=0, \quad 0 \leqslant x \leqslant 1
$$

and the conditions

$$
\left.\frac{\partial u}{\partial t}\right|_{x=0}-\left.\frac{\partial u}{\partial x}\right|_{x=1}=h_{1}(t),\left.\quad \frac{\partial u}{\partial x}\right|_{x=0}+\left.\frac{\partial u}{\partial t}\right|_{x=1}=h_{2}(t),
$$

are satisfied. Here $h_{1}$ i $h_{2}$ are given continuous functions on $[0,1 / 2]$ such that $h_{1}(0)=h_{2}(0)=0$.

All the assumptions of Theorem 1 hold, except for condition (7), since it is easy to verify that $\operatorname{det} \alpha_{1}(t) \equiv 0$ in this case.

It can be easily checked that any continuously differentiable solution of (38) on $G$ satisfying initial conditions (39) is given by

$$
u(x, t)= \begin{cases}f(t-x), & 0 \leqslant x \leqslant t \\ 0, & t \leqslant x \leqslant 1-t, \quad t \in[0,1 / 2], \\ g(t+x-1), & 1-t \leqslant x \leqslant 1,\end{cases}
$$


where $f(t)$ and $g(t)$ are arbitrary continuously differentiable functions on $[0,1 / 2]$ that satisfy the conditions $f(0)=f^{\prime}(0)=g(0)=g^{\prime}(0)=0$. By $(40)$, we come to

$$
f^{\prime}(t)-g^{\prime}(t) \equiv h_{1}(t), \quad-f^{\prime}(t)+g^{\prime}(t) \equiv h_{2}(t) \text {. }
$$

Therefore, problem (38)-(40) is solvable if and only if $h_{2}(t)=-h_{1}(t)$. If this condition is fulfilled, then

$$
g(t)=f(t)-\int_{0}^{t} h_{1}(\tau) d \tau,
$$

and the function $f$ remains arbitrary. Thus, the problem above is either unsolvable or has an infinite set of solutions. Hence, the estimate (9) is impossible.

Now let us seek the solution of (38) on $G$ satisfying initial conditions (39) and the conditions

$$
\begin{aligned}
& \left.\frac{\partial u}{\partial t}\right|_{x=0}-\left.\frac{\partial u}{\partial x}\right|_{x=1}=h_{1}(t), \\
& \int_{0}^{1}(1-2 \xi) \frac{\partial u(\xi, t)}{\partial t} d \xi=h_{2}(t),
\end{aligned}
$$

where $h_{1}(t)$ and $h_{2}(t)$ are given continuous functions on $[0,1 / 2]$ such that $h_{1}(0)=h_{2}(0)=0$, and, in addition, the function $h_{2}$ is continuously differentiable.

We see that all the conditions of Theorem 3 are satisfied except for condition (22), since in the case under consideration $\operatorname{det} \beta(t) \equiv 0$.

By requiring that (41) again satisfy conditions (43), we conclude that problem (38), (39), (43) is solvable if and only if $h_{1}$ and $h_{2}$ are connected by

$$
h_{2}(t)=\int_{0}^{t}(1-2(t-\tau)) h_{1}(\tau) d \tau .
$$

If this condition is satisfied, then the problem has an infinite set of solutions of the form (41), where $f$ is arbitrary function, and $g$ has the form (42).

It is easy to build similar examples for cases of failure of condition (19) or (36).

Acknowledgements. The authors dedicate this paper to the memory of Z. O. Melnyk (1935-1983), our Teacher, who interested us in the study of boundary-value problems for hyperbolic equations and systems, and gave us his experience and knowledge. The idea of this paper belongs to him.

\section{REFERENCES}

1. Abolinya V.̇̀., Myshkis A.D. On a mixed boundary-value problem for a linear hyperbolic system on a plane// Uch. Zap. Latv. Univ. - 1958. - V.XX, №3. - P. 87-104.

2. Akhmedova A.N., Namazov G.K. Mixed problem for a second-order hyperbolic equation with discontinuous coefficients// Izv. Akad. Nauk AzSSR. Ser. Fiz.-Tekhn. i Mat. Nauk. - 1965. - №2. - P. 3-11.

3. Andrusyak R.V., Kirilich V.M., Myshkis A.D.P. Local and global solvability of the vquasilinear hyperbolic Stefan problem on the line// Diff. Equations. - 2006. - V.42, №4. - P. 519-536.

4. Babenko K.I., Gelfand I.M. Notes on hyperbolic systems// Nauchn. Dokl. Vys. Shkoly. Fiz.-Mat. Nauki. 1958. - №1. - P. 12-18. 
5. Berehova H.I. Hyperbolic Stefan problem with nonlocal boundary conditions// Visn. Lviv. Univ. Ser. Mech.-Mat. - 1996. - №48. - P. 104-112.

6. Berehova H.I., Kyrylych V.M. Hyperbolic Stefan problem in a curvilinear sector// Ukr. Math. J. - 1997. V.49. - P. 1899-1906. https://doi.org/10.1007/BF02513068. Transl. from Ukr. Mat. Zhurn. - 1997. V.49, №12. - P. 1684-1689.

7. Brazma N.A. On solving by grid method of a simplest mixed problem for matrix telegraph equation// Izv. Akad. Nauk Latv. SSR. - 1956. - V.3. - P. 133-138.

8. Brazma N.A. Review of research on the system of matrix telegraph equations performed in Riga// Izv. Akad. Nauk Latv. SSR. - 1958. - V.8. - P. 133-141.

9. Budak B.M. On homogeneous second order of accuracy differential-difference schemes for parabolic and hyperbolic equations with discontinuous coefficients // Dokl. Akad. Nauk SSSR. - 1962. - V.142, №5. P. 986-989.

10. Campbell L.L., Robinson A. Mixed problems for hyperbolic partial differential equations// Proc. London Math. Soc. - 1955. - №18. - P. 129-147.

11. Citrini C., d'Acuto B. Sur le chic de deux cordes// C.R. Acad. sci. - 1979. - V.289, №1.

12. Courant R. Partial differential equations. - M: Mir, 1964. - 830 p., Transl. from Courant R., Hilbert D. Methods of Mathematical Physics, V.2.

13. Dennis I.E., Traub I.F., Weber R.P. The algebraic theory of matrix polynomials // SIAM I Numer. Anal. 1976. - V.13. - P. 831-845.

14. Dragieva N.A. Solution of a hyperbolic equation in a domain with moving boundaries// Godyshn. Vyssh. Uchebn. Zaved. Prilozh. Mat. - 1977. - V.11, №2. - P. 53-64.

15. Filimonov A.M., Lapin D.S. Mixed problem for singular quasilinear hyperbolic systems with a single spatial variable// Mat. Zametki. - 2003. - V.73, №2. - P. 315-318.

16. Filimonov A.M., Myshkis A.D. Continuous solutions of hyperbolic systems of quasilinear equations with two independent variables// Nelin. Anal. i Nelin. Diff. Uravn. - M.: Fiz. Mat. Lit., 2003.- P. 337-351.

17. Firman T., Kyrylych V. Mixed problem for countable hyperbolic system of linear equation// Azerbaijan Journal of Mathematics. - 2015. - V.5, №2. - P. 47-60.

18. Florescu D.D. Asupra existentei solutiolor unor sisteme hiperbolice de tip special// Stud. si cerc. mat. 1978. - V.30, №3. - P. 279-285.

19. Gasanov K.K. Fourier method for a one-dimensional quasilinear hyperbolic equation with discontinuous coefficients // Uch. Zap. Azerb. Univ. Ser. Fiz.-Mat. Nauki. - 1966. - №1. - P. 9-16.

20. Goldberg M., Tadmor E. Scheme-independent stability criteria for difference approximations of hyperbolic initial-boundary value problems. I// Mathematics of Computation. - 1978. - V.32, №144. - P. 10971107.

21. Gustaffson B., Kreiss H.-O., Sundström A. Stability theory of difference approximations for mixed initialboundary value problems. II // Mathematics of Computation. - 1972. - V.26, №119. - P. 649-686.

22. Il'kiv V.S., Symotyuk M.M., Volyanska I.I. Nonlocal boundary-value problem for a second-order partial differential equation in an unbounded strip// Ukr. Mat. J. - 2019. - V.70, №10. - P. 1585-1593.

23. Kabulov V.K. Study of oscillations of constant cross section beams using balance-type integral equations // Vychisl. Mat. - 1958. - №3. - P. 138-148.

24. Kalenyuk P.I., Volyanska I.I., Il'kiv V.S., Nytrebych Z.M. On the unique solvability of a three-point problem for partial differential equation in a two-dimensional domain// J. Math. Sci. - 2020. - V.246, №2. - P. 170-187.

25. Khmelev D.V., Oseledets V.I. Global stability of infinite systems of nonlinear differential equations and nonhomogeneous countable Markov chains// Probl. Peredachi Inf. - 2000. - V.36. - №1. - P. 60-76. English version: Oseledets V.I., Khmelev D.V. Global stability of infinite systems of nonlinear differential equations and nonhomogeneous countable Markov chains, Problems Inform. Transmission. - 2000. - V.36, №1. - P. 54-70.

26. Kmit I.Ya. Nonlocal boundary value problems for hyperbolic systems of equations with singularities: author's abstract of the doctor-degree thesis in phys.-mat. sciences. - Kyiv, 2013. - 36p.

27. Kovalev V.A. On a new method of reconstruction solutions of a boundary value problem for an hyperbolic type equation// Diff. i Integr. Uravn. - Gorkiy, 1981. - P. 125-130.

28. Kreiss H.-O. Difference approximations for the initial-boundary value problems for hyperbolic differential equations// Numer. Solut. Nonlinear Different. - New York-London-Sydney: John. Willey and Sons. 1966. - P. 141-166. 
29. Kyrylych V.M., Melnik Z.O. Problems without initial conditions with integral restrictions for hyperbolic equations and systems on a line// Ukr. Mat. J. - 1983. - V.35, №6. - P. 722-727.

30. Kyrylych V.M. A nonclassical problem with integral restrictions for a first-order two-dimensional hyperbolic system// Visn. Lviv. Univ. Ser. Mech. Mat. - 1983. - №21. - P. 60-64.

31. Kyrylych V.M. Hyperbolic Stefan problem with nonlocal boundary conditions for the system of quasilinear equations // Mat. Stud. - 2009. - V.32, №2. - P. 216-221.

32. Kyrylych V.M. Free-boundary problems for hyperbolic systems of first-order quasilinear partial differential equations: author's abstract of the doctor-degree thesis in phys.-mat. sciences. - Kyiv, 2010. - 32p.

33. Ladyzhenskaya O.A. On application of the method of finite differences to the solution of the Cauchy problem for hyperbolic systems// Dokl. Akad. Nauk SSSR. - 1953. - V.88, №4. - P. 607-610.

34. Ladyzhenskaya O.A. Mixed Cauchy problem for hyperbolic equation. - M: Nauka, 1953. - 280p.

35. Ladyzhenskaya O.A. Application of functional analysis to existence proof of solutions to boundary value problems for partial differential equations of three main types// Uspekhi Mat. Nauk. - 1955. - V.10, №4. - P. 200-202.

36. Ladyzhenskaya O.A., Vishik M.I. Boundary value problems for partial differential equations and certain classes of operator equations // Uspekhi Mat. Nauk. - 1956. - V.11, №6. - P. 41-97.

37. Lenkov V.M. Solution of boundary value problem for hyperbolic equation in domain with moving boundaries// Tr. Mosc. Vyssh. Tekhn. Uchil. im N. E. Baumana. - 1977. - V.256. - P. 50-56.

38. Magal P., Ruan S. Senter manifolds for semilinear equations with nondence domain and applications to Hopf bifurcation in age structured models// Mem. Am. Math. Soc. - 2009. - V.951. - P. 1-71.

39. Mamedov K.M. The solution of the mixed problem for one general one-dimensional hyperbolic equation// Tr. Inst. Fiz. i Mat. Akad. Nauk AzSSR. Ser. Mat. - 1955. - V.7. - P. 33-54.

40. Markovsky A.I. Regularizers of mixed problems for hyperbolic systems on the plane// Dokl. Akad. Nauk USSR. - 1973. - V.A, №12. - P. 1083-1086.

41. Markovsky A.I. Regularizers of mixed problems for hyperbolic systems on the plane// Mat. Fiz.: Sb. Nauch. Tr. - Kiev, 1974. - Issue. 16. - P. 124-132.

42. Markovsky A.I. Regularizers of mixed problems for hyperbolic systems on finite interval// Kraevye zadachi mat. fiz. - Kiev, 1979. - P. 124-138.

43. Markovsky A.I. On the regularizers of some mixed problems on a plane// Mat. Fiz.: Sb. Nauch. Tr. Kiev, 1979. - Issue. 25. - P. 100-106.

44. Melnik Z.O. Mixed problem for general third- and fourth-order hyperbolic equations on a plane// Dokl. Akad. Nauk USSR. - 1963. - №9. - P. 1149-1151.

45. Melnik Z.O. On some general mixed problem// Dokl. Akad. Nauk SSSR. - 1964. - V.157, №5. - P. 1039 1042.

46. Melnik Z.O., Myshkis A.D. Mixed problem for a two-dimensional first-order hyperbolic system with discontinuous coefficients// Mat. Sb. - 1965. - V.68, №4. - P. 632-638.

47. Melnik Z.O. Mixed problem for general second-order hyperbolic equation on a plane// Dokl. Akad. Nauk USSR. - 1965. - №4. - P. 419-422.

48. Melnik Z.O. On one way to solve the mixed problem for a hyperbolic equation with discontinuous coeffcients // Diff. Uravn. - 1966. - №4. - P. 560-570.

49. Melnik Z.O. General mixed problems for general two-dimensional hyperbolic systems// Diff. Uravn. 1966. - №7. - P. 958-966.

50. Melnik Z.O. On some integro-differential equation in a compound domain// Sib. Mat. J. - 1966. - V.7, №3. - P. 577-590.

51. Melnik Z.O. On hyperbolic equations with multiple characteristics// Diff. Uravn. - 1974. - V.10, №8. P. $1530-1532$.

52. Melnik Z.O. Example of a nonclassical boundary-value problem for the equations of vibrations of a string// Ukr. Mat. J. - 1980. - V.32, №5. - P. 671-673.

53. Melnik Z.O. One non-classical boundary value problem for a first-order hyperbolic system with two independent variables// Diff. Uravn. - 1981. - V.17, №6. - P. 1096-1104.

54. Myshkis A.D., Shlopak A.S. Mixed problem for systems of partial differential equations and Volterra-type operators// Mat. Sb. - 1957. - V.41, №2. - P. 239-256.

55. Namazov V.M. On solvability of a one-dimensional mixed problem for a Kovalevskaya system with discontinuous coefficients and an expansion formula associated with it// Uch. Zap. Azerb. Univ. Ser. Fiz.-Mat. Nauk. - 1966. - №6. - P. 18-27. 
56. Namazov V.M. Necessary conditions for solvability of a mixed problem for a first-order Kovalevskaya system// Uch. Zap. Azerb. Univ. Ser. Fiz.-Mat. Nauk. - 1972. - №4. - P. 45-49.

57. Nahushev A.M. Boundary value problems for loaded integro-differential equations of hyperbolic type and some of their applications to soil moisture forecasting// Diff. Uravn. - 1979. - V.15, №1. - P. 69-105.

58. Nahushev A.M. Loaded equations and their applications// Diff. Uravn. - 1983. - V.19, №1. - P. 86-96.

59. Nastaj B. A hyperbolic Stefan problem in vacuum freeze drying of disordered porous media// Bull. Pol. Acad. Sci. Tech. Sci. - 2000. - V.48, №3. - P. 357-368.

60. Peyser G. Energy integral for the mixed problem in hyperbolic differential equations of higher order// J. Math. and Mech. - 1957. - V.6, №5. - P. 641-653.

61. Peyser G. The Goursat problem for hyperbolic equations// J. Math. and Mech. - 1961. - V.10, №1. P. 91-109.

62. Phillips R.S. Dissipative hyperbolic systems// Trans. Amer. Math. Soc. - 1957. - V.86, №1. - P. 109-173.

63. Ptashnyk B.Yo., Ilkiv V.S., Kmit I.Ya., Polishchuk V.M. Nonlocal boundary value problems for partial differetial equations. - K.: Naukova dumka, 2002. - 415p.

64. Rasulov M.L. Application of the contour integral method, M.: Nauka, 1975. - 255p.

65. Sanchez D.A. Linear age-dependent population growth with harvesting// Bull. Biol. - 1978. - V.40, №3. - P. 377-385.

66. Shum L.F. On one way to solve a telegraph equation with discontinuous coefficients// Izv. Akad. Nauk BSSR. Ser. Fiz.-Mat. Nauk. - 1966. - №1. - P. 36-45.

67. Sinha D.K. A note on mechanical response in a pieso electric transducer owing to an impulsive voltage input// Proc. Nat. Inst. India. - 1966. - V.31, №4. - P. 395-402.

68. Sobolevsky P.E., Pogorelenko V.A. On solvability of mixed problems for one-dimensional quasilinear hyperbolic equations// Ukr. Mat. J. - 1970. - V.22, №1. - P. 114-121.

69. Strikwerda J.C. Initial boundary value problems for the methods of lines // J. Comput. Phys. - 1980. V.34, №1. - P. 94-107.

70. Thomee V. Estimates of the Friedrichs-Lewy type for mixed roblems in the theory of linear hyperbolic differential equations in two independent variables // Math. Scand. - 1957. - V.5, №1. - P. 93-113.

71. Thomee V. Existence proof for mixed roblems for hyperbolic differential equations in two independent variables by means of continuity method// Math. Scand. - 1958. - V.6, №1. - P. 5-32.

72. Thomee V. Difference methods for two-dimensional mixed roblems for hyperbolic first order systems // Arch. Ration. Mech. and Analysis. - 1961. - V.8, №1. - P. 68-88.

73. Thomee V. A mixed boundary-value problem for hyperbolic first order systems with derivatives in the boundary conditions // Arch. Ration. Mech. and Analysis. - 1961. - V.8, №5. - P. 435-443.

74. Thomee V. A prodictor-corrector type scheme for the mixed boundary problem for a hyperbolic first order system in two dimensions// J. Soc. Industr. and Appl. Math. - 1963. - V.11, №4. - P. 964-975.

75. Vagabov A.I. Solution of one-dimensional mixed problems for a first-order hyperbolic system// Uch. Zap. Azerb. Univ., Ser. Fiz.-Mat. Nauki. - 1963. - №4. - P. 11-17.

76. Vagabov A.I. Conditions for the correctness of one-dimensional mixed problems for hyperbolic systems// Dokl. Akad. Nauk SSSR. - 1964. - V.155, №6. - P. 1247-1249.

77. Winther R.A. A stable finite element method for initial-boundary value problems for first-order hyperbolic systems // Math. Comput. - 1981. - V.36, №153. - P. 65-86.

78. Zacharias V. Ein spezialles Goursat-Problem in der Ebene// Monatsber. Deutsch. Akad. Wiss. - Berlin, 1969. - V.11, №8-9. - P. 685-695.

79. Zhdanovich V.F. Solution by the Fourier method of non-self-adjoint mixed problems for hyperbolic systems on a plane// Mat. Sb. - 1959. - V.47, №3. - P. 307-354.

Ivan Franko National University of Lviv,

Lviv, Ukraine

vkyrylych@ukr.net

Lviv Polytechnic National University,

Lviv, Ukraine

olga_slusarchuk@ukr.net 